\title{
Calcium co-Localization with in vivo Cerium Phosphate Nanoparticle Formation after Intratracheal Instillation Dosing with $\mathrm{CeCl}_{3}$ or $\mathrm{CeO}_{2} \mathrm{NPs}$
}

\author{
Uschi M. Graham ${ }^{1}$, Joseph Fernback ${ }^{2}$, Chen Wang ${ }^{3}$, Alan K. Dozier ${ }^{1}$, Lawrence Drummy ${ }^{2}$, \\ Oberdörster $^{4}$ and Joseph D. Brain ${ }^{3}$ \\ 1. National Institute for Occupational Safety and Health, Cincinnati, OH, USA \\ 2. Air Force Research Laboratory, Dayton, OH, USA \\ ${ }^{3 .}$ Harvard T.H. Chan School of Public Health, Boston, MA, USA \\ 4. University of Rochester, Rochester, NY, USA
} Krishnamurthy Mahalingam ${ }^{2}$, Ramon M. Molina ${ }^{3}$, Nagarjun V. Konduru ${ }^{3}$, M. Eileen Birch ${ }^{1}$, Günter

The bioprocessing of $\mathrm{CeO}_{2}$ nanoparticles after uptake in lung tissues is compared with the formation of nanoparticles after inhalation of $\mathrm{CeCl}_{3}$ aerosols. In both cases, high-resolution TEM/STEM analyses indicate that cerium phosphate nanoparticles (NPs) had precipitated in phagolysosomal regions within macrophages. Importantly, the primary particle size, morphology, and agglomeration tendencies of the NPs were strikingly similar. Formation of cerium phosphate NPs after bioprocessing of inhaled $\mathrm{CeO}_{2}$ crystals proceeds via dissolution, ion transport, followed by nucleation and growth [1]. Application of 2D and 3D elemental maps of the NP bioprocessing stages and corresponding tissue interactions provide insights on the breakdown mechanisms, formation of new precipitates, size, and morphology changes of the original delivered NPs, and ion transport phenomena that result in secondary particle formation. Particle size and morphology are very similar for all of the cerium phosphate NPs, suggesting a common underlying precipitation mechanism independent of whether the metal ions are derived from dissolution of nanoparticles $\left(\mathrm{CeO}_{2}\right)$ or from instilled metal ions $\left(\mathrm{CeCl}_{3}\right)$.

In the lungs, bioprocessing of $\mathrm{CeO}_{2}$ and $\mathrm{CeCl}_{3}$ resulted in the formation of rod-shaped, aligned particles that were identified in EELS and EDS analyses as cerium phosphate (Figure 1). Surprisingly, elemental analyses also reveal for the first time, and with excellent reproducibility, the presence of $\mathrm{Ca}$ in the same localities as the in vivo precipitated cerium phosphate NPs (Figure 2). What controls the preferential coprecipitation, or what underlying pathways control the localized $\mathrm{Ca}$ enrichment are questions to be answered.

Lung tissue samples were fixed by vascular perfusion with $2.5 \%$ glutaraldehyde, $2 \%$ paraformaldehyde in 0.1M HEPES buffer. The samples were processed for electron microscopy at 2 hours, 7 days, and 4 weeks post-instillation. High resolution imaging revealed that NPs formed at all of the selected timepoints and were located in lysosomal regions, along membrane surfaces, and inside mitochondria. The NPs had formed in a narrow particle size range $(\sim 1-5 \mathrm{~nm})$ and were bundled together in 3D-agglomerates that ranged up to several hundred $\mathrm{nm}$ (Figure 1). At high magnification, the individual nanoparticles tended to self-align into rod-shaped structures.

\section{References:}

[1] U.M. Graham et al, ChemPlusChem 79(8) (2014), p. 1083.

[2] R.M. Molina et al, Environ. Sci.: Nano 1 (2014), p. 561. 


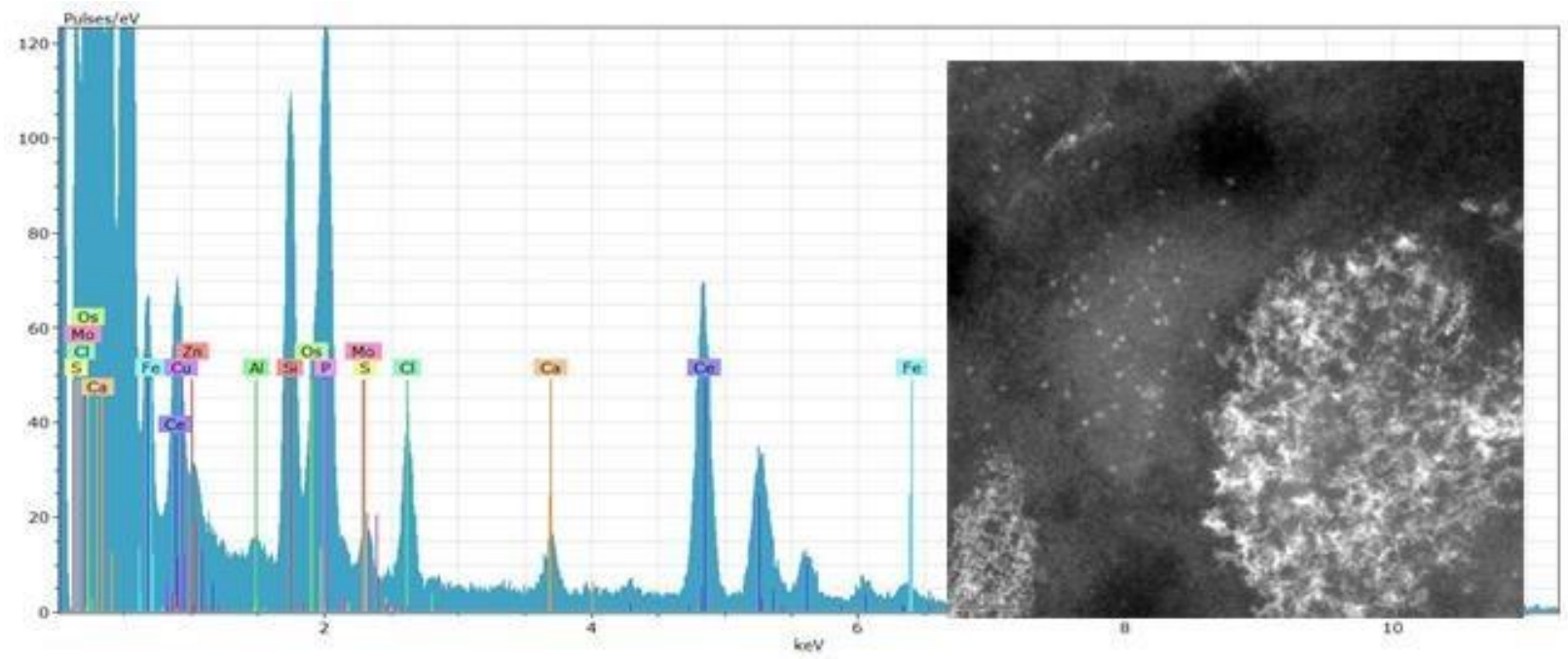

Figure 1. STEM image showing precipitated cerium phosphate nanoparticles as agglomerates in lung tissue. The corresponding 2D area EDS analysis indicates the presence of calcium $(\mathrm{Ca})$.
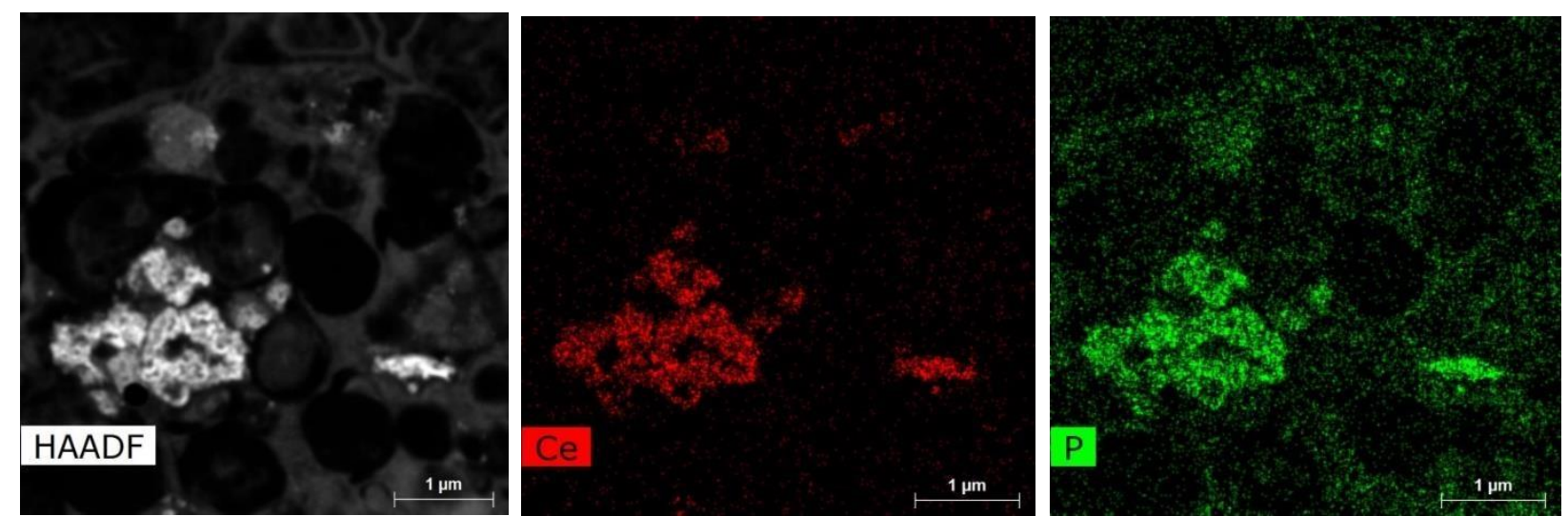

Figure 2.

HAADF image of precipitated cerium phosphate nanoparticles inside lung tissue is indicated by the $\mathrm{Ce}, \mathrm{P}, \mathrm{O}$ and $\mathrm{P}$ elemental maps. Calcium is clearly enriched in the areas where significant CeP nanoparticle formation
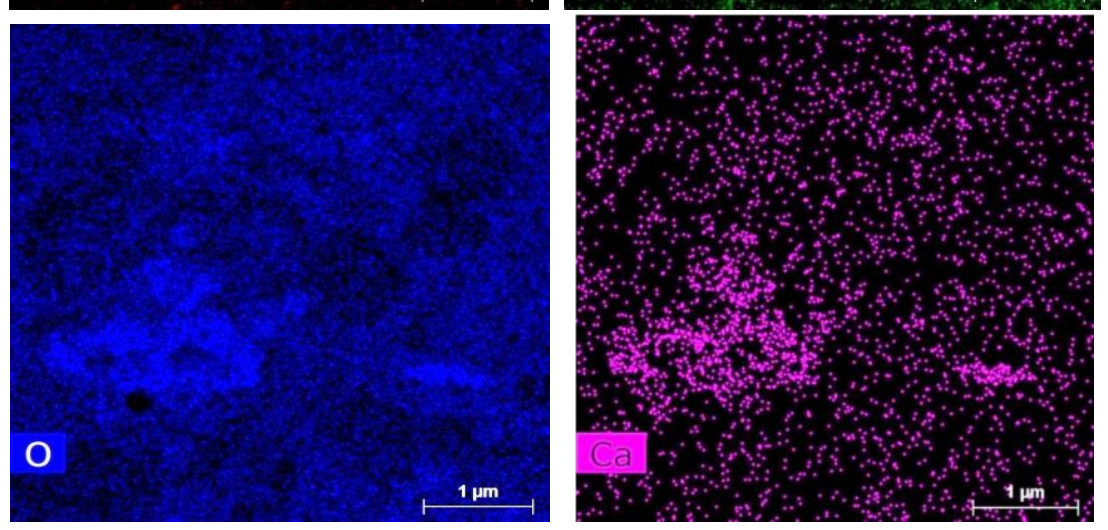